\title{
CATACLYSMIC VARIABLES FROM THE SLOAN DIGITAL SKY SURVEY. VIII. THE FINAL YEAR (2007-2008)*
}

\author{
Paula Szkody ${ }^{1}$, Scott F. Anderson ${ }^{1}$, Keira Brooks ${ }^{1}$, Boris T. Gänsicke ${ }^{2}$, Martin Kronberg $^{1}$, \\ Thomas Riecken $^{1}$, Nicholas P. Ross ${ }^{3}$, Gary D. Schmidt ${ }^{4}, 11$, Donald P. Schneider ${ }^{5}$, Marcel A. Agüeros ${ }^{6}$, \\ Ada N. GomeZ-Moran ${ }^{7,8}$, Gillian R. Knapp ${ }^{9}$, Matthias R. Schreiber ${ }^{10}$, and Axel D. Schwope ${ }^{7}$ \\ ${ }^{1}$ Department of Astronomy, University of Washington, Seattle, WA 98195, USA; szkody@ astro.washington.edu \\ ${ }^{2}$ Department of Physics, University of Warwick, Coventry CV4 7AL, UK \\ ${ }^{3}$ Lawrence Berkeley National Lab, Berkeley, CA 92420, USA \\ ${ }^{4}$ The University of Arizona, Steward Observatory, Tucson, AZ 85721, USA \\ ${ }^{5}$ Department of Astronomy and Astrophysics, Pennsylvania State University, University Park, PA 16802, USA \\ ${ }^{6}$ Department of Astronomy, Columbia University, New York, NY 10027, USA \\ ${ }^{7}$ Leibniz-Institut für Astrophysik Potsdam (AIP), 14482 Potsdam, Germany \\ ${ }^{8}$ CNRS, Observatoire Astronomique de Strasbourg, 67000 Strasbourg, France \\ ${ }^{9}$ Princeton University Observatory, Princeton, NJ 08544, USA \\ ${ }^{10}$ Departamento de Física y Astronomía, Universidad de Valparaíso, Chile \\ Received 2011 June 7; accepted 2011 September 17; published 2011 October 20
}

\begin{abstract}
This paper completes the series of cataclysmic variables (CVs) identified from the Sloan Digital Sky Survey (SDSS) I/II. The coordinates, magnitudes, and spectra of $33 \mathrm{CVs}$ are presented. Among the 33 are eight systems known prior to SDSS (CT Ser, DO Leo, HK Leo, IR Com, V849 Her, V405 Peg, PG1230+226, and HS0943+1404), as well as nine objects recently found through various photometric surveys. Among the systems identified since the SDSS are two polar candidates, two intermediate polar candidates, and one candidate for containing a pulsating white dwarf. Our follow-up data have confirmed a polar candidate from Paper VII and determined tentative periods for three of the newly identified CVs. A complete summary table of the 285 CVs with spectra from SDSS I/II is presented as well as a link to an online table of all known CVs from both photometry and spectroscopy that will continue to be updated as future data appear.
\end{abstract}

Key words: binaries: close - binaries: spectroscopic - catalogs - novae, cataclysmic variables - stars: dwarf novae

Online-only material: machine-readable and VO tables

\section{INTRODUCTION}

Since the beginning of the Sloan Digital Sky Survey (SDSS; York et al. 2000), we have analyzed the available spectra to produce a listing of cataclysmic variables (CVs) in the growing database each calendar year. This paper completes the listing for the last year of the primary SDSS I-II surveys and the data available in the final SDSS I-II public release (DR7; Abazajian et al. 2009 ${ }^{12}$ ). The previous listings of CVs are available in Papers I-VII (Szkody et al. 2002, 2003, 2004, $2005,2006,2007,2009)$. As in the previous papers, the CVs include dwarf novae, nova-like variables, and objects with highly magnetic white dwarfs (polars and intermediate polars (IPs)). These types of CVs are reviewed in detail in Warner (1995).

The value of the SDSS-selected objects is that they provide a large sample of CVs with spectra of medium resolution $(\sim 3 \AA)$

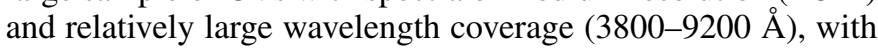
accurate coordinates, finding charts, and photometry available for all objects. This has led to many follow-up observations by a multitude of groups that resulted in the determination of over a hundred orbital periods. With this significant amount of information, the viability of CV population models could be explored. Prior to DR7, Gänsicke et al. (2009) used the

\footnotetext{
* Based on observations obtained with the Sloan Digital Sky Survey and with the Apache Point Observatory (APO) $3.5 \mathrm{~m}$ telescope, which are owned and operated by the Astrophysical Research Consortium (ARC).

${ }^{11}$ Currently at NSF.

12 Data are available online from http://www.sdss.org.
}

available results for 116 systems to show that the increased depth of SDSS provided a new window on the period distribution. The periods from the SDSS CVs were much closer to the predictions of population models (Howell et al. 2001) than previous surveys with brighter limits that better sampled long period systems. The final 33 objects in this paper provide a small (10\%) increment to the total number of SDSS CVs, but complete the work of the last decade and present several interesting systems for future study. In addition to the new objects, we also include follow-up polarimetry on a polar from Paper VII (SDSSJ093839.25+534403.8).

\section{OBSERVATIONS AND REDUCTIONS}

The specific details concerning the SDSS survey can be found in Pier et al. (2003), Gunn et al. (1998, 2006), Lupton et al. (1999), Hogg et al. (2001), Lupton et al. (2001), Ivezic et al. (2004), Smith et al. (2002), Tucker et al. (2006), and Padmanabhan et al. (2008). The methods of detecting the CVs are stated in Szkody et al. (2002) and in the publications on the selection algorithms (Stoughton et al. 2002; Richards et al. 2002). As a very brief summary, the objects in the imaging data obtained in $u, g, r, i, z$ filters (Fukugita et al. 1996) are selected for spectral fibers through the use of established color algorithms, with galaxies and quasars receiving the bulk of the fibers for spectra. Fortunately, the colors of quasars and hot objects cover many of the CVs and so most of the acquired spectra come from QSO candidates (see Richards et al. 2002 for a description of the QSO selection algorithm and Gänsicke et al. 2009 for details of the QSO color selection overlap with CVs). 
Table 1

CVs in SDSS

\begin{tabular}{|c|c|c|c|c|c|c|c|c|}
\hline SDSS J & MJD-P-F ${ }^{a}$ & $g$ & $u-g$ & $g-r$ & $r-i$ & $i-z$ & $\begin{array}{c}P \\
(\mathrm{hr})\end{array}$ & Comments $^{\mathrm{b}}$ \\
\hline $034420.16+093006.8$ & 54368-2679-299 & 18.19 & -0.28 & 0.00 & 0.03 & 0.00 & $\cdots$ & \\
\hline $035409.32+100924.4$ & 54389-2697-028 & 20.89 & 0.38 & -0.09 & -0.03 & -0.16 & $\cdots$ & \\
\hline $073208.11+413008.7$ & $54154-2702-411$ & 20.77 & -0.31 & 0.32 & 0.20 & 0.39 & $\cdots$ & DN, W \\
\hline $075648.04+305805.0$ & $54537-2959-046$ & 21.07 & -0.10 & 0.10 & -0.08 & -0.10 & $\cdots$ & CSS \\
\hline $075653.11+085831.8$ & $54505-2945-144$ & 16.27 & 0.42 & 0.00 & -0.07 & -0.04 & 2 & \\
\hline $091242.18+620940.1$ & $54450-1786-573$ & 18.81 & -0.06 & 0.07 & 0.29 & 0.57 & 2 & DN, W \\
\hline $092809.83+071130.5$ & $54169-2382-502$ & 15.32 & 0.25 & -0.17 & -0.16 & -0.20 & $\cdots$ & $\mathrm{DN}, \mathrm{W}$ \\
\hline $092918.90+622346.2$ & $54465-1787-052$ & 18.72 & 0.53 & 0.29 & 0.04 & 0.13 & $\cdots$ & \\
\hline $093220.92+133122.2$ & $54093-2578-620$ & 18.02 & 0.24 & -0.08 & -0.17 & -0.07 & $\cdots$ & \\
\hline $094634.46+135057.8$ & $54139-2582-185$ & 17.11 & -0.23 & 0.10 & 0.00 & 0.00 & 4.2 & IP HS $0943+1404$ \\
\hline $095151.79+471008.7$ & $54525-2956-373$ & 20.32 & 0.04 & 0.00 & -0.29 & 0.19 & $\cdots$ & \\
\hline $104051.23+151133.6$ & $54117-2594-026$ & 17.96 & 0.28 & 0.42 & 0.36 & 0.16 & 5.63 & DO Leo \\
\hline $100516.61+694136.5$ & $54478-1879-608$ & 19.41 & 0.32 & 0.48 & 0.31 & 0.34 & $\cdots$ & IP?, W \\
\hline $105550.08+09560.4$ & 54498-2886-504 & 19.20 & -0.14 & 0.60 & 0.60 & 0.50 & $\cdots$ & $\mathrm{DN}, \mathrm{CSS}$ \\
\hline $111703.53+182558.1$ & $54453-2857-085$ & 14.64 & -0.40 & -0.25 & -0.01 & 0.04 & 42.2 & HK Leo \\
\hline $112003.40+663632.4$ & $54498-2858-239$ & 15.65 & 0.18 & -0.24 & -0.23 & -0.20 & $\cdots$ & $\mathrm{DN}, \mathrm{W}$ \\
\hline $112253.31-111037.6$ & $54561-2874-603$ & 20.47 & 0.22 & 0.01 & -0.12 & 0.13 & $\cdots$ & CSS \\
\hline $120724.69+223529.8$ & $54210-2644-431$ & 19.98 & 0.22 & 0.7 & 0.47 & 0.00 & $\cdots$ & polar? \\
\hline $121913.04+204938.3$ & $54477-2611-376$ & 19.17 & 0.26 & -0.03 & -0.16 & -0.12 & $\cdots$ & \\
\hline $122405.58+184102.7$ & $54477-2611-042$ & 16.01 & 0.08 & -0.03 & -0.18 & -0.13 & $\cdots$ & \\
\hline $123255.11+222209.4$ & $54495-2647-200$ & 17.70 & -0.10 & -0.15 & -0.09 & -0.11 & $\cdots$ & PG1230+226 \\
\hline $123932.00+210806.2$ & $54481-2613-523$ & 18.33 & -0.09 & -0.08 & 0.37 & 0.78 & 2.09 & IR Com \\
\hline $131709.07+245644.2$ & $54234-2663-583$ & 19.09 & 0.42 & 0.69 & 0.63 & 0.29 & $\cdots$ & \\
\hline $132856.71+310846.0$ & $53467-2110-480$ & 17.61 & -0.48 & -0.16 & -0.34 & -0.29 & $\cdots$ & \\
\hline $134441.83+204408.3$ & $54231-2654-023$ & 17.17 & -0.12 & -0.72 & -0.38 & -0.07 & 2 & polar? \\
\hline $151500.56+191619.6$ & $54271-2793-450$ & 17.83 & -0.36 & -0.43 & -0.27 & -0.07 & $\cdots$ & \\
\hline $151915.86+064529.1$ & $54562-1834-417$ & 16.58 & -0.05 & -0.16 & -0.14 & -0.05 & $\cdots$ & \\
\hline $154539.07+142231.6$ & $54567-2517-581$ & 16.33 & 0.04 & -0.11 & -0.14 & -0.07 & 4.68 & CT Ser \\
\hline $161027.61+090738.4$ & $54582-2526-098$ & 20.13 & 0.07 & 0.04 & -0.18 & 0.28 & $\cdots$ & $\mathrm{DN}, \mathrm{W}$ \\
\hline $162207.15+192236.6$ & $54589-2970-466$ & 18.63 & -0.20 & 0.48 & -0.05 & 0.23 & $\cdots$ & \\
\hline $162520.29+120308.7$ & $54572-2531-446$ & 18.51 & -0.06 & 0.09 & 0.16 & 0.56 & 2.19 & DN, W \\
\hline $163545.72+112458.0$ & 54585-2533-156 & 15.21 & 0.32 & -0.13 & -0.11 & -0.10 & 3.4 & V849 Her \\
\hline $230949.12+213516.7$ & $54328-2623-193$ & 16.10 & -0.32 & 0.74 & 0.70 & 0.56 & 4.26 & V405 Peg \\
\hline
\end{tabular}

Notes.

${ }^{\text {a }}$ MJD-Plate-Fiber for spectra; MJD = JD - 2,400,000.5.

b DN is a dwarf nova, W is Wils et al. (2010), CSS is Drake et al. (2009).

Software programs as well as sporadic eye searches are then used to identify objects with zero redshifts and Balmer emission/absorption lines that could be CVs. Additionally, discrepancies in magnitude between the photometry and spectroscopy can be an indication of an object that has transitioned from outburst to quiescence or from low to high states of mass transfer. Thus, the selection is not complete but from comparisons of known CVs from past catalogs (Ritter \& Kolb 2003) in areas covered by the SDSS, comparison of our manual searches to computer findings, and comparison of CV colors to those of QSOs (Gänsicke et al. 2009), we estimate that the completeness of blue CVs with $i \leqslant 19.1 \mathrm{mag}$ is roughly similar to that for ultraviolet excess quasars ( $\sim 90 \%$; Schneider et al. 2007).

Table 1 lists the 33 objects in DR7 from 2007 January 1 to the conclusion of SDSS II in 2008 June. As in the previous papers, this table shows the plate, fiber, and modified Julian Date (MJD) of the spectra, along with the coordinates in equinox J2000.0 (truncated to the last digit) with an accuracy of 0.10 arcsec. Magnitudes and colors are obtained using the point-spread function (PSF) photometry, with no correction for reddening. For the rest of this paper, we will simplify the SDSS names to SDSSJhhmm (hours and minutes of R.A.) except for SDSS0745, which needs the first digits of declination as there are two objects with similar abbreviated R.A.s.
Table 2

Follow-up APO Spectra

\begin{tabular}{lcccc}
\hline \hline SDSSJ & UT Date & $\begin{array}{c}\text { Time } \\
(\mathrm{UT})\end{array}$ & $\begin{array}{c}\text { Exp } \\
(\mathrm{s})\end{array}$ & Number of Spectra \\
\hline $0756+08$ & 2010 May 5 & $02: 47-04: 15$ & 600 & 8 \\
0912 & 2009 Mar 29 & $02: 20-04: 48$ & 600 & 12 \\
0929 & 2009 Apr 18 & $03: 43-04: 38$ & 600 & 5 \\
0929 & 2010 May 5 & $04: 56-06: 39$ & 600 & 9 \\
$0938^{\text {a }}$ & 2009 Feb 27 & $02: 43-07: 08$ & 600 & 22 \\
1055 & 2010 May 5 & $04: 31$ & 600 & 1 \\
1344 & 2009 Apr 18 & $05: 10-06: 56$ & 600 & 9 \\
\hline
\end{tabular}

Note. ${ }^{a}$ Object discovered in Paper VII.

Follow-up observations were obtained for a few objects using the Apache Point Observatory (APO) $3.5 \mathrm{~m}$ telescope and the Dual Imaging Spectrograph with high-resolution gratings $(2 \AA)$ and a 1.5 arcsec slit. One set of spectra was also accomplished for the possible polar presented in Paper VII (SDSSJ0938). A summary of these spectral follow-up observations is given in Table 2. Spectropolarimetry of SDSSJ0938 was also obtained on the $2.3 \mathrm{~m}$ Bok telescope with the CCD Spectropolarimeter on 2008 October 29 (4800 s) and 2009 April 26 (3600 s), and 
Table 3

SDSS Emission Line Fluxes and Equivalent Widths ${ }^{\mathrm{a}}$

\begin{tabular}{|c|c|c|c|c|c|c|c|c|}
\hline \multirow[t]{2}{*}{ SDSSJ } & \multicolumn{2}{|c|}{$\mathrm{H} \beta$} & \multicolumn{2}{|c|}{$\mathrm{H} \alpha$} & \multicolumn{2}{|c|}{$\mathrm{He} 4471$} & \multicolumn{2}{|c|}{ He II 4686} \\
\hline & $\mathrm{F}$ & EW & $\mathrm{F}$ & EW & $\mathrm{F}$ & EW & $\mathrm{F}$ & EW \\
\hline 0344 & 2.7 & 10 & 2.9 & 16 & 0.7 & 2 & $\ldots$ & $\ldots$ \\
\hline 0354 & 0.1 & 9.5 & 0.4 & 53 & $\ldots$ & $\ldots$ & $\ldots$ & $\ldots$ \\
\hline 0732 & 0.6 & 23 & 0.8 & 29 & $\ldots$ & $\ldots$ & $\ldots$ & $\ldots$ \\
\hline $0756+30$ & 0.9 & 69 & 1.1 & 136 & 0.2 & 17 & $\ldots$ & $\ldots$ \\
\hline $0756+08$ & 11.7 & 23 & 10.6 & 35 & 4.1 & 7 & 9.9 & 18.5 \\
\hline 0912 & 2.6 & 44 & 4.9 & 124 & $\ldots$ & $\ldots$ & $\ldots$ & $\ldots$ \\
\hline 0928 & $\ldots$ & $\ldots$ & $\ldots$ & $\ldots$ & $\ldots$ & $\ldots$ & $\ldots$ & $\ldots$ \\
\hline 0929 & 1.4 & 9 & 1.6 & 14 & 0.5 & 3 & 0.6 & 3.6 \\
\hline 0932 & $\ldots$ & $\ldots$ & 1.1 & 8.5 & $\ldots$ & $\ldots$ & $\ldots$ & $\ldots$ \\
\hline 0946 & 5.7 & 34 & 14.2 & 48 & 4.4 & 8 & 12.7 & 24 \\
\hline 0951 & 1.7 & 76 & 2.5 & 243 & $\ldots$ & $\ldots$ & $\ldots$ & $\ldots$ \\
\hline 1005 & 7.7 & 43 & 6.2 & 46 & 2.1 & 11 & 5.1 & 29 \\
\hline 1040 & 13.5 & 4.3 & 23.3 & 15 & $\ldots$ & $\ldots$ & 21.0 & 6 \\
\hline 1055 & 14.9 & 3 & 22.8 & 9 & $\ldots$ & $\ldots$ & $\ldots$ & $\ldots$ \\
\hline 1117 & 23.3 & 4 & 24.5 & 9 & 5.3 & 0.6 & 11.8 & 2 \\
\hline 1120 & 0.8 & 0.9 & 10 & $\ldots$ & $\ldots$ & $\ldots$ & $\ldots$ & \\
\hline 1122 & 0.5 & 24 & 0.9 & 87 & 0.3 & 10 & 0.1 & 4 \\
\hline 1207 & 1.2 & 35 & 1.2 & 45 & 0.5 & 16 & 0.7 & 20 \\
\hline 1219 & 1.6 & 22 & 3.7 & 87 & $\ldots$ & $\ldots$ & $\ldots$ & $\ldots$ \\
\hline 1224 & 5.6 & 4 & 6.4 & 9 & $\ldots$ & $\ldots$ & $\ldots$ & $\ldots$ \\
\hline 1232 & 1.7 & 5 & 2.3 & 13 & $\ldots$ & $\ldots$ & 1.2 & 3 \\
\hline 1239 & 41.6 & 106 & 59.1 & 222 & $\ldots$ & $\ldots$ & $\ldots$ & $\ldots$ \\
\hline 1317 & 1.2 & 12 & 1.8 & 13 & 0.2 & 2 & $\ldots$ & $\ldots$ \\
\hline 1328 & 15.3 & 33 & 49.7 & 142 & 1.2 & 2 & $\ldots$ & $\ldots$ \\
\hline 1344 & 1.1 & 7 & 1.4 & 19 & $\ldots$ & $\ldots$ & $\ldots$ & $\ldots$ \\
\hline 1515 & 1.2 & 3 & 1.0 & 6 & $\ldots$ & $\ldots$ & $\ldots$ & $\ldots$ \\
\hline 1519 & 4.3 & 4 & 7.3 & 16 & $\ldots$ & $\ldots$ & 2.0 & 2 \\
\hline 1545 & 5.7 & 5 & 6.2 & 10 & 1.1 & 0.7 & 4.1 & 3 \\
\hline 1610 & 0.6 & 19 & 1.3 & 76 & $\ldots$ & $\ldots$ & $\ldots$ & $\ldots$ \\
\hline 1622 & 14.1 & 100 & 12.9 & 111 & 3.1 & 20 & 0.8 & 6 \\
\hline 1625 & 1.6 & 33 & 3.5 & 111 & $\ldots$ & $\ldots$ & $\ldots$ & $\ldots$ \\
\hline 1635 & $\ldots$ & $\ldots$ & 3.5 & 2 & $\ldots$ & $\ldots$ & $\ldots$ & \\
\hline 2309 & 85.4 & 82 & 122.6 & 71 & 15.4 & 16 & $\ldots$ & $\ldots$ \\
\hline
\end{tabular}

Note. ${ }^{\text {a }}$ Fluxes are in units of $10^{-15} \mathrm{erg} \mathrm{cm}^{-2} \mathrm{~s}^{-1}$, equivalent widths are in units of $\AA$.

on the $1.5 \mathrm{~m}$ Kuiper telescope on 2009 January 30, 31 (2400 s each night) and February 1 (1600 s).

The calibrations for flux and wavelength for the APO data, as well as the line measurements, were accomplished with standard IRAF ${ }^{13}$ routines. The " $e$ " (centroid) routine in the IRAF splot package was used to measure the SDSS spectra for line centers, equivalent widths, and fluxes of the Balmer and helium emission lines. Lines were measured several times to estimate an error on the measured velocities. The fluxes and equivalent widths are listed in Table 3. The solutions for the radial velocity curves for the time-resolved spectra were obtained by using a least-squares fit of a sine curve to the velocities to determine $\gamma$ (systemic velocity), $K$ (semi-amplitude), $P$ (orbital period), and $T_{0}$ (the UT time of red to blue crossing of the systemic velocity). A Monte Carlo method was used to generate the errors on $\gamma$ and $K$. These solutions are listed in Table 4 along with the standard deviation $(\sigma)$ of the sine fit to the data points. Since the APO observation times are typically allotted in half-nights, the periods are not well established. The period uncertainties are typically $10 \%$ based on differences between the $\mathrm{H} \beta$ and $\mathrm{H} \alpha$ lines and should only be used as a basis for classification of an object as under or

\footnotetext{
13 IRAF (Image Reduction and Analysis Facility) is distributed by the National Optical Astronomy Observatory, which is operated by AURA, Inc., under cooperative agreement with the National Science Foundation.
}

over the period gap that exists in the distribution of CVs between 2 and $3 \mathrm{hr}$.

\section{RESULTS}

Figure 1 shows the SDSS spectra of the 33 objects in Table 1. Details on several classes of systems are presented below.

\subsection{Previously Known Systems}

There are eight objects in Table 1 that were known as CVs prior to the SDSS. SDSSJ1545 is Nova Ser 1948 (CT Ser) which has been studied by Ringwald et al. (2005) who determined an orbital period of 0.195 days. Four objects were identified as blue sources in the Palomar-Green survey (Green et al. 1986). SDSSJ1040 is PG1030+155 (DO Leo) which Abbott et al. (1990) identified as an eclipsing nova-like variable with an orbital period of $5.63 \mathrm{hr}$. The SDSS spectrum presented in Figure 1 has a greater strength of He II than shown in Abbott et al. (1990). The ratio of $\mathrm{He} \mathrm{II} / \mathrm{H} \beta$ in the SDSS spectrum is 1.4 (Table 3) while this ratio is 0.33 in Abbott et al. (1990). SDSSJ1117 is PG1114+187 (HK Leo), identified as a pre-CV with an orbital period of 1.76 days by Hillwig et al. (2000). SDSSJ1232 is PG1230+226, which has no further information available. The fourth object, SDSSJ1635, is PG1633+115 (V849 Her) and was studied photometrically by Misselt \& Shafter 

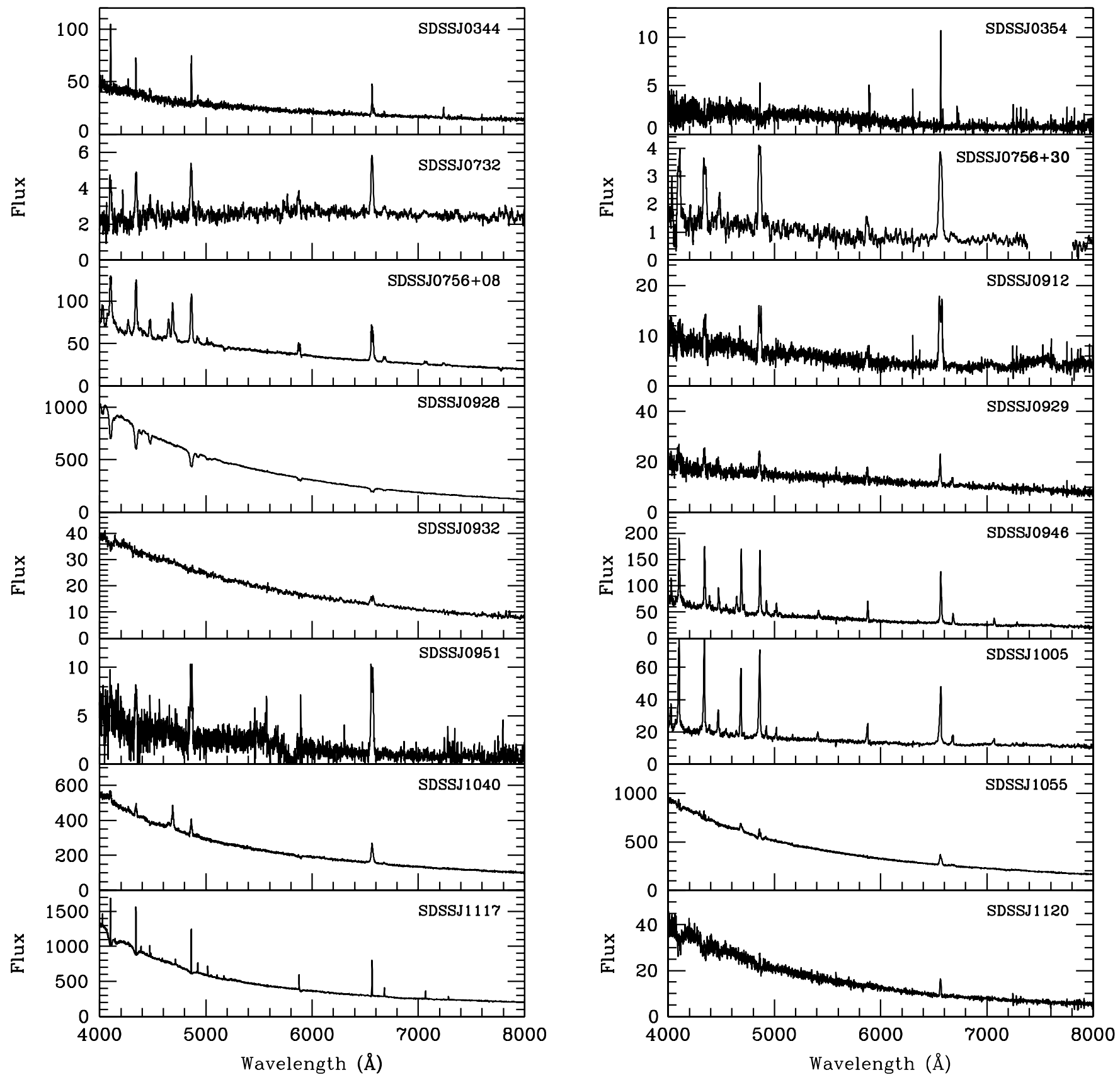

Figure 1. SDSS spectra of the $33 \mathrm{CVs}$. Vertical axis is units of flux density $F_{\lambda} \times 10^{-17} \mathrm{erg} \mathrm{cm}^{-2} \mathrm{~s}^{-1} \AA^{-1}$. The spectral resolution is about $3 \AA$. The gaps in SDSSJ0756+30 (between 7400 and $7800 \AA$ ) and SDSSJ1239 (between 4000 and $4800 \AA$ ) are due to unusable sections of the data.

Table 4

Radial Velocity Solutions

\begin{tabular}{lcccccc}
\hline \hline SDSSJ & Line & $\begin{array}{c}P \\
(\text { minutes })^{\mathrm{a}}\end{array}$ & $\begin{array}{c}\gamma \\
\left(\mathrm{km} \mathrm{s}^{-1}\right)\end{array}$ & $\begin{array}{c}K \\
\left(\mathrm{~km} \mathrm{~s}^{-1}\right)\end{array}$ & $\begin{array}{c}T_{0} \\
(\mathrm{UT})\end{array}$ & $\begin{array}{c}\sigma \\
\left(\mathrm{km} \mathrm{s}^{-1}\right)\end{array}$ \\
\hline $0756+08$ & $\mathrm{H} \alpha$ & 117 & $241 \pm 5$ & $166 \pm 16$ & $4: 15$ & 24 \\
$0756+08$ & $\mathrm{H} \beta$ & 110 & $192 \pm 7$ & $129 \pm 28$ & $4: 11$ & 44 \\
0912 & $\mathrm{H} \alpha$ & $113^{\mathrm{b}}$ & $1.1 \pm 2.5$ & $40 \pm 8$ & $4: 04$ & 17 \\
0912 & $\mathrm{H} \beta$ & 113 & $22 \pm 3$ & $60 \pm 9$ & $4: 21$ & 18 \\
1344 & $\mathrm{H} \alpha$ & 122 & $34 \pm 1$ & $374 \pm 15$ & $5: 32$ & 27 \\
1344 & $\mathrm{H} \beta$ & 110 & $47 \pm 2$ & $412 \pm 23$ & $5: 33$ & 42 \\
\hline
\end{tabular}

Notes.

${ }^{a}$ Periods are generally uncertain by $10 \%$, as evidenced by the dispersion between values obtained from the two lines.

${ }^{\mathrm{b}}$ Due to low $K$ and large sigma, best fit provided by fixing the period to that determined from $\mathrm{H} \beta$. 

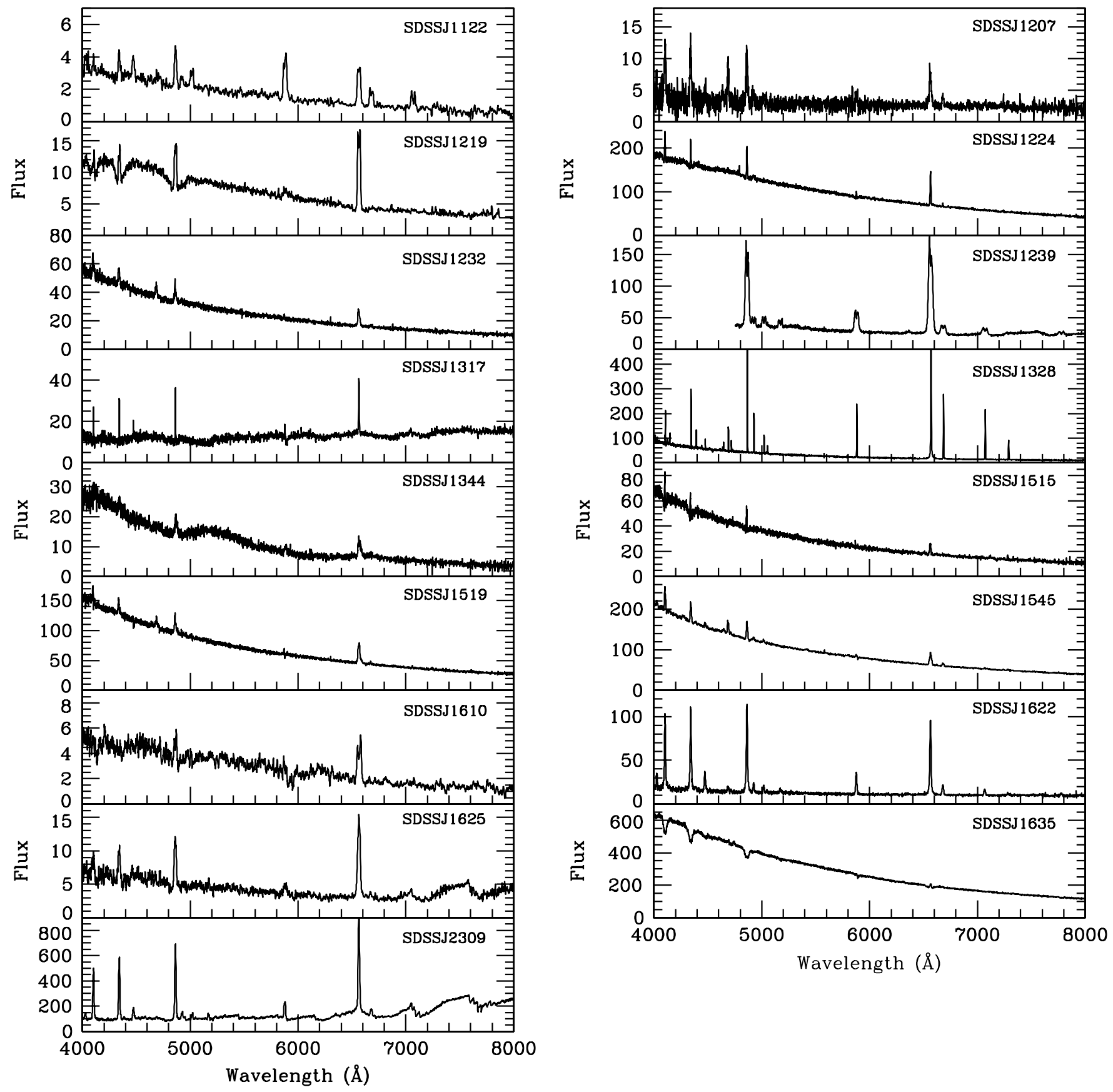

Figure 1. (Continued)

(1995) who determined a period near 203 minutes but could not sort out aliases with their data. The Hamburg Quasar survey (Hagen et al. 1995) first found HS0943+1404 which is SDSSJ0946. Rodriguez-Gil et al. (2005) identified this variable as an IP with a spin period of 69 minutes and an orbital period near $4.2 \mathrm{hr}$. The last two objects were found as counterparts to bright objects in the ROSAT survey. IR Com (SDSSJ1239) was discovered by Richter \& Greiner (1995) and later found to be an eclipsing dwarf nova with an orbital period of 125.3 minutes (Wenzel et al. 1995); further detailed photometry was accomplished by Feline et al. (2005). V405 Peg is SDSSJ2309, discovered by Schwope et al. (2002), and recently studied in detail by Thorstensen et al. (2009), who found it to have an unusually low accretion rate for a system with an orbital period of $4.26 \mathrm{hr}$ and suggested that it could be a system in hibernation. Their spectra show states of some accretion (strong emission lines) and states of lower accretion (weak emission). The SDSS spectrum in Figure 1 appears similar to those of slightly enhanced accretion.

Recently, Wils et al. (2010) performed a correlation of GALEX, the Catalina Sky Survey (CSS; Drake et al. 2009), and SDSS photometry as well as a search for multiple SDSS photometric observations that showed variability, resulting in a set of CV candidates. Included in this group of likely dwarf novae are SDSSJ0732, SDSSJ0756+30, SDSSJ0912, SDSSJ0928, SDSSJ1005, SDSSJ1610, SDSSJ1625, and SDSSJ1120. Olech et al. (2011) recently determined an orbital period of $131.2 \mathrm{~min}$ utes for SDSSJ1625 following an outburst of this system. Two further systems (SDSSJ1055 and SDSSJ1122) were identified as CV candidates by the CSS and appear on the CSS Web site 

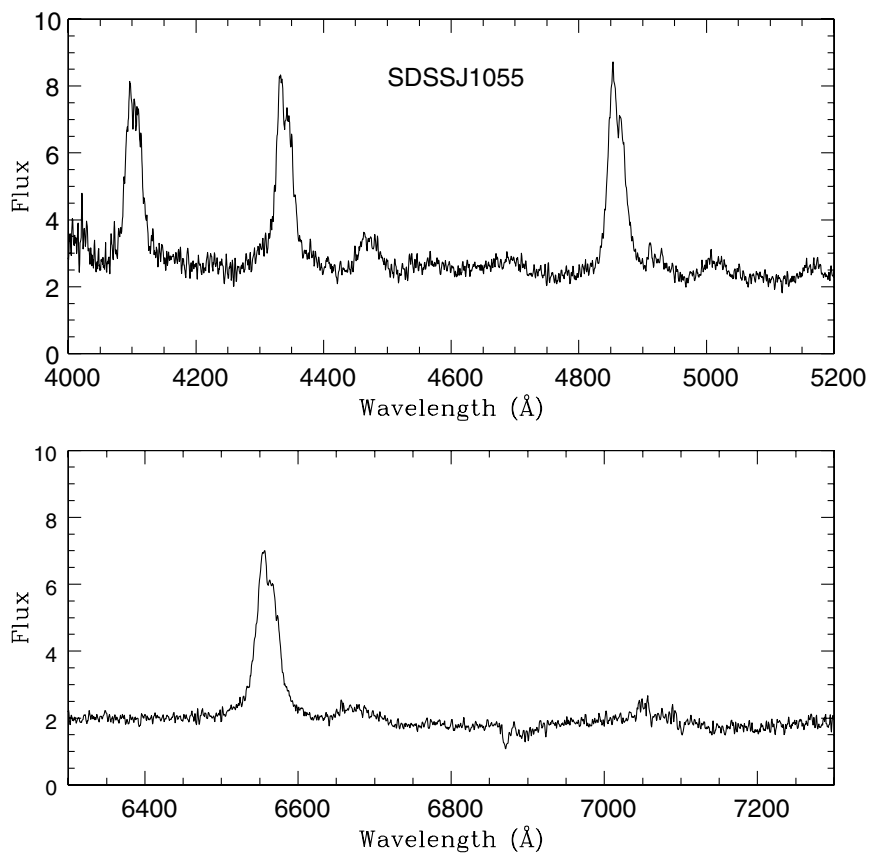

Figure 2. APO blue and red spectra of SDSSJ1055 at quiescence. Vertical axis is flux density $F_{\lambda} \times 10^{-16} \mathrm{erg} \mathrm{cm}^{-2} \mathrm{~s}^{-1} \AA^{-1}$.

of $\mathrm{CVs}^{14}$ (Drake et al. 2009). SDSSJ1122 was followed up by vsnet during an outburst (Maehara 2010a, 2010b; Kato 2010), with superhumps at a period of 65.4 minutes reported.

\subsection{Dwarf Novae}

Dwarf novae are recognized by their outbursts, revealed by several magnitude increases in photometric measurements as well as by the spectral changes from Balmer emission to absorption lines during the outburst. The different times of SDSS photometry and spectroscopy allow some dwarf novae to be identified. Past United States Naval Observatory and Digitized Sky Survey catalogs and ongoing CSS photometry also provide chances to detect outbursts. Wils et al. (2010) and Drake et al. (2009) used these methods to provide nine dwarf nova candidates. Comments on the SDSS spectra and our follow-up observations for several of these systems are given below.

SDSSJ0928 has strikingly different SDSS photometric and spectroscopic magnitudes (the spectrum in Table 1 was obtained at outburst) and SDSSJ1120 has two SDSS spectra, one at outburst and one at quiescence.

The spectrum of SDSSJ1055 shown in Figure 1 is at outburst, while the SDSS photometry reports a $g$ mag of 19.2. An APO spectrum obtained at quiescence (Table 2 ) is shown in Figure 2. The visibility of a sharp peak component in the lines indicates a prominent hot spot may contribute to the light of this system.

SDSSJ0912. The pronounced doubling of the Balmer lines in this object (Figure 1) indicates a relatively high inclination. Time-resolved spectra over $2.3 \mathrm{hr}$ of this system during quiescence produced radial velocity curves (Figure 3 ) indicating a period near $2 \mathrm{hr}$. Table 4 lists the best fits for the $\mathrm{H} \beta$ and $\mathrm{H} \alpha$ lines, which involve a relatively low $K$ semi-amplitude. However, the relatively poor quality of the fit requires a longer data string to produce a reliable solution.

\footnotetext{
14 http://nesssi.cacr.caltech.edu/catalina/AllCV.html
}

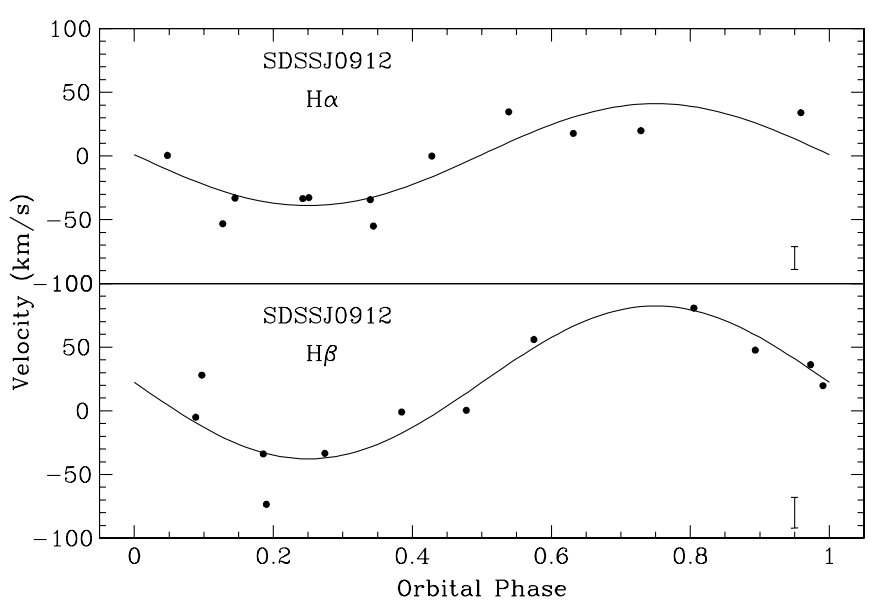

Figure 3. $\mathrm{H} \alpha$ and $\mathrm{H} \beta$ velocity curves of SDSSJ0912 from the APO data. The smooth curves are the best-fit sinusoids (Table 4). Sigmas of the sine fits are listed in Table 4. Estimates of the observed error on the measured velocities is shown by the error bars near phase 0.9 .

\subsection{Polars}

Polars are identified spectroscopically either from the presence of strong He II together with large amplitude radial velocity curves for these lines or from cyclotron harmonics (Wickramasinghe \& Ferrario 2000). The former is usually present for systems in high states of accretion, while cyclotron humps are most visible for systems with low accretion (e.g., Schmidt et al. 2005). In both cases, the observation of circular polarization provides the confirmation of a polar. Figure 1 shows the spectra of our three prime candidates for polars, SDSSJ1005, SDSSJ1207, and SDSSJ1344. The first two have the strong He II lines of a high state, while SDSSJ1344 reveals a single cyclotron hump. It is possible that SDSS1005 may be an IP, as its spectrum is similar to the identified IP SDSSJ0946 (Figure 1). Polarization observations will determine the correct identification. The follow-up observations of SDSSJ1344 and a polar candidate from Paper VII (SDSSJ0938) are discussed below.

SDSSJ0938. In Paper VII, SDSSJ0938 was identified as a $g=19.15 \mathrm{mag}$ polar candidate, with the spectrum showing strong He II. We have subsequently obtained spectropolarimetry when the system was at a similar magnitude (2008 October 29) and also on four nights in 2009 January-April when the system was in a low state (about a magnitude fainter). Time-resolved APO spectra over $4.2 \mathrm{hr}$ were also obtained in 2009 February during the faint state.

The results of the spectropolarimetry are presented in Figure 4. The circular polarization was measured to be weak but significant and always showed positive values. There are several puzzling aspects to these data. The presence of circular polarization confirms that this is a system with a magnetic white dwarf. As the polarization is present throughout the optical spectrum, the field must be relatively strong, and the lack of cyclotron harmonics rules out a low-mass accretion system. However, it is not clear why the polarization is so much smaller than in normal polars and why the value does not change when the system goes into the low state and the emission lines disappear.

The time-resolved APO spectra provided few clues. Whereas seven spectra over 70 minutes obtained during the high state in 2008 (Paper VII) showed a smooth variation during this interval, the $4.2 \mathrm{hr}$ during the low state showed only a constant velocity with a standard deviation of $19 \mathrm{~km} \mathrm{~s}^{-1}$. Only the 


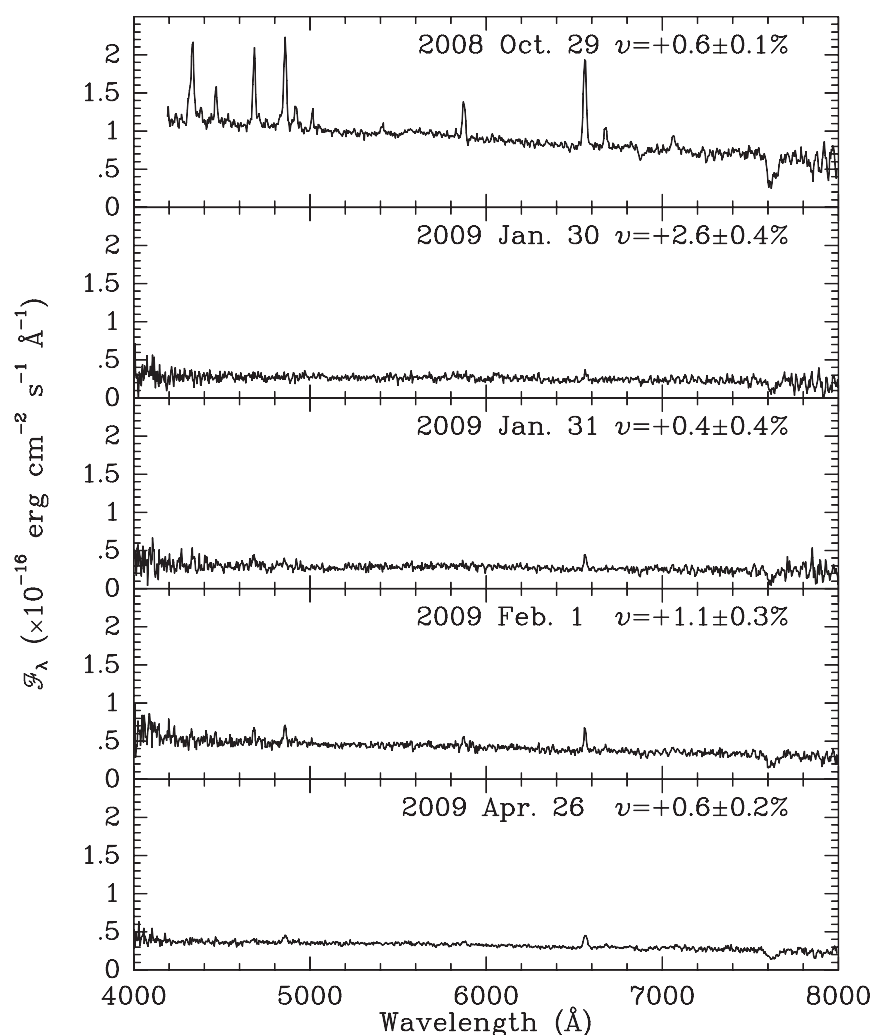

Figure 4. Spectra and polarization values of SDSSJ0938 for five nights in 2008-2009 during high and low states.

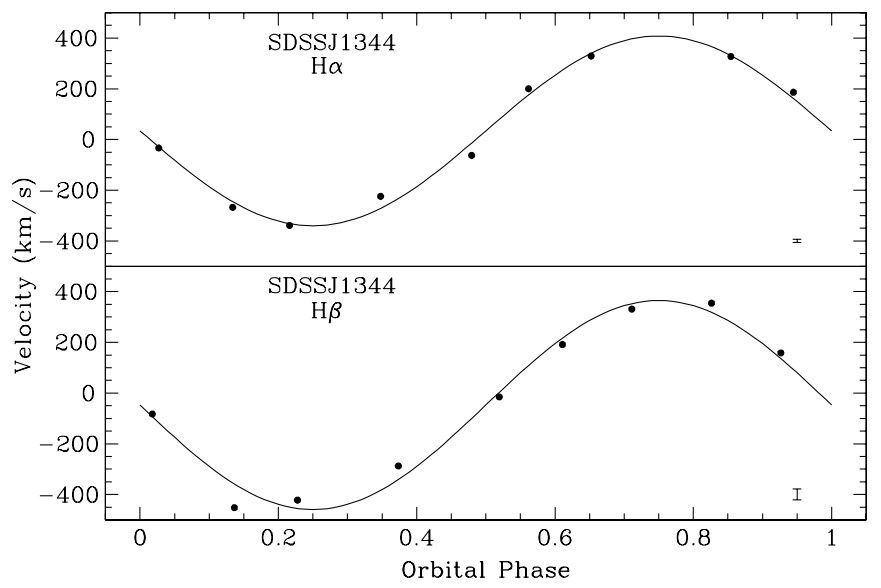

Figure 5. $\mathrm{H} \alpha$ and $\mathrm{H} \beta$ velocity curves of SDSSJ1344 from APO data with the best-fit sinusoids from Table 4 superposed. Estimates of the measured velocities are shown by the error bars near phase 0.9 .

$\mathrm{H} \alpha$ line was strong enough to measure. Further data when the system re-enters a higher state are needed to resolve the physical characteristics of this system.

SDSSJ1344. While the Balmer emission lines of SDSSJ1344 are relatively weak, the time-resolved APO spectra were able to show a large radial velocity variation during the 107 minutes of observation. Figure 5 shows the radial velocity curves while Table 4 lists the best fits. The large $K$ amplitude confirms the likely polar nature, but polarimetry will be required to obtain the field strength as the single cyclotron hump visible in Figure 1 does not provide enough information to establish which harmonic is viewed.

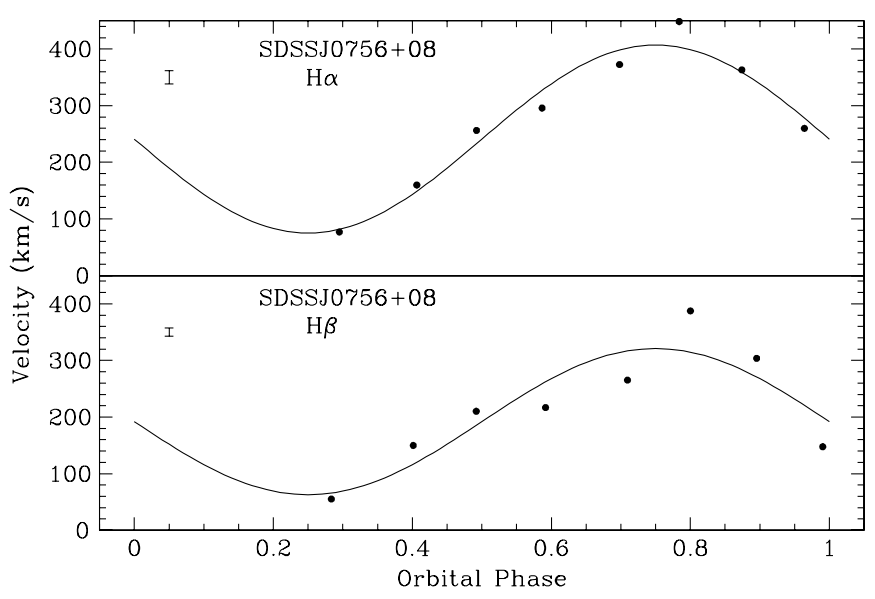

Figure 6. $\mathrm{H} \alpha$ and $\mathrm{H} \beta$ velocity curves of SDSSJ0756+08 from APO data with the best-fit sinusoids from Table 4 superposed. Estimates of the error on the measured velocities are shown near phase 0.05 .

\subsection{Nova-likes with He II}

Besides the previously identified IP (SDSSJ0946), the nova CT Ser (SDSSJ1545), the eclipsing nova-like DO Leo (SDSSJ1040), and the outbursting dwarf nova (SDSSJ1055), the other objects in Figure 1 that have significant He II emission are SDSSJ0756+08, SDSSJ1122, SDSSJ1232, and SDSSJ1519. Of these four CVs, SDSSJ1232 and SDSSJ1519 display the strong blue continuum and weak emission lines consistent with a high accretion rate nova-like system. The other two objects have strong Balmer lines. SDSSJ1122 is particularly interesting in that the He II line is weak but the He I lines are unusually strong. Unfortunately, follow-up observations could be obtained only for SDSSJ0756+08.

SDSSJ0756+08. The SDSS spectrum (Figure 1) resembles that of the IP SDSSJ0946. Although our coverage is only $1.5 \mathrm{hr}$, there is an obvious sinusoidal modulation apparent indicating a period near 2 hr. Figure 6 shows the radial velocity curves and Table 4 presents the solution with our limited data. If this short period and possible IP nature are confirmed, this object would fall into the small class of IPs with periods under the gap. ${ }^{15}$

\subsection{Systems Showing the Underlying Stars}

As reported in Papers I-VII, the systems with lowest accretion rates allow the underlying stars to be visible, as evidenced by broad Balmer absorption lines from the white dwarf surrounding the disk emission lines and/or the presence of TiO bands from a late type secondary. In Figure 1, SDSSJ0912, SDSSJ1317, SDSSJ1625, and SDSSJ2309 show evidence for an $M$ star. SDSSJ1219 shows the clear signature of a white dwarf in a low accretion system, and SDSSJ1610 may be in this category. Since several of the white dwarfs with similar spectra have been shown to undergo non-radial pulsations, SDSSJ1219 should have high priority for time-resolved photometry. SDSSJ0354, SDSSJ1224, SDSSJ1317, and SDSSJ1515 have absorption with very narrow emission lines. This group could be pre-CVs with the emission caused by irradiation from the secondary by a hot white dwarf.

\subsection{Other Disk Systems}

SDSSJ0932 shows a blue continuum with only a broad double-peaked emission line of $\mathrm{H} \alpha$ (Figure 1). Since the

\footnotetext{
15 See http://asd.gsfc.nasa.gov/Koji.Mukai/iphome/iphome.html for a listing of all known IPs.
} 
Table 5

ROSAT Detections

\begin{tabular}{lcrlc}
\hline \hline SDSSJ & $\begin{array}{c}\text { ROSAT } \\
\left(\text { counts s }^{-1}\right)^{\mathrm{a}}\end{array}$ & $\begin{array}{c}\text { Exp } \\
(\mathrm{s})\end{array}$ & & RXS \\
\hline 0946 & $0.027 \pm 0.011$ & 345 & RXS J094635.9+135126 & Type \\
0951 & $0.0076 \pm 0.0020$ & 2712 & RXP J095152.1+471004 & IP \\
1005 & $0.048 \pm 0.018$ & 243 & RXS J100518.3+694101 & $\ldots$ \\
1117 & $0.0023 \pm 0.0006$ & 23865 & WGA J1117.0+1826= HK Leo & IP? \\
1239 & $0.061 \pm 0.013$ & 431 & RXS J123930.6+210815= IR Com & DN \\
2309 & $0.212 \pm 0.022$ & 466 & RXS J230949.6+213523 = V405 Peg & NL \\
\hline
\end{tabular}

Notes. ${ }^{\text {a }}$ For a $2 \mathrm{keV}$ bremsstrahlung spectrum, 1 counts s${ }^{-1}$ corresponds to a $0.1-2.4 \mathrm{keV}$ flux of about $7 \times 10^{-12} \mathrm{erg} \mathrm{cm}^{-2} \mathrm{~s}^{-1}$.

photometric magnitudes are similar in brightness to the spectroscopic fluxes, this is not a dwarf nova in outburst but likely a high accretion rate disk system (a nova-like variable).

\subsection{ROSAT Correlations}

Six of the 33 systems were detected in X-ray with the ROSAT All Sky Survey (Voges et al. 1999, 2000). Table 5 gives the detected count rates and exposure times for those CVs. Of the three candidate polars, only SDSSJ1005 (the possible IP) is detected. It is not too surprising that all three candidates are not detected as the others could have been in low accretion states (common for polars) at the time of the ROSAT observations.

\section{SUMMARY}

The addition of these 33 systems brings the total spectroscopically identified CVs in SDSS to 285. The objects deserving of particular attention are the polar candidates SDSSJ1207 and SDSSJ1344, the possible IPs SDSSJ0756 and SDSSJ1005, the object with unusual He I abundance (SDSSJ1122), and the candidate for a system containing a pulsating white dwarf (SDSSJ1219).

A summary table of the 285 objects that have SDSS spectra from Papers I-VIII is presented here (Table 6). This table has 22 columns that provide the object name, R.A. and decl. in degrees, $u, g, r, i, z$ PSF magnitudes and errors, MJD, plate and fiber of the spectroscopic observation, MJD of the photometric observation, the spectroscopic target selection flags (detailed in Stoughton et al. 2002), the period if known, the discovery reference, the type of system, and any comments. In addition, an active Web list of these objects, with links to the spectra and information on types and period is being kept and updated (as new information becomes available). ${ }^{16}$ If an outburst is observed, a classification as dwarf nova can be made, and as spectroscopic/photometric studies appear in the literature, further orbital periods will become known. At the present time, there are 151 periods known for these CVs, with 98 (65\%) under the gap $(<2 \mathrm{hr})$. The list of objects includes 30 polars (with seven being very low accretion rate polars or LARPs), six IPs, and nine that contain pulsating white dwarfs. While the SDSS sample is not $100 \%$ complete due to the selection effects discussed in Gänsicke et al. (2009), this database will provide a wealth of information for statistical studies of spectra and characteristics of CVs for years to come. In addition, future observations of the peculiar systems which do not seem to fit the prescribed mold of currently known CVs can provide some useful information on the evolution that occurs in close binaries.

\footnotetext{
$\overline{16}$ http://www.astro.washington.edu/users/szkody/cvs/index.html
}

Table 6

SDSS I/II CV Catalog Format

\begin{tabular}{lcl}
\hline \hline Column & Format & \multicolumn{1}{c}{ Description } \\
\hline 1 & A18 & SDSS Designation hhmmss.ss+ddmmss.s (J2000) \\
2 & F11.6 & R.A. (J2000) in degrees \\
3 & F11.6 & Decl. (J2000) in degrees \\
4 & F7.3 & $u$ PSF magnitude \\
5 & F6.3 & Error in $u$ PSF magnitude \\
6 & F7.3 & $g$ PSF magnitude \\
7 & F6.3 & Error in $g$ PSF magnitude \\
8 & F7.3 & $r$ PSF magnitude \\
9 & F6.3 & Error in $r$ PSF magnitude \\
10 & F7.3 & $i$ PSF magnitude \\
11 & F6.3 & Error in $i$ PSF magnitude \\
12 & F7.3 & $z$ PSF magnitude \\
13 & F6.3 & Error in $z$ PSF magnitude \\
14 & I6 & MJD of spectroscopic observation \\
15 & I5 & Plate of spectroscopic observation \\
16 & I4 & Fiber of spectroscopic observation \\
17 & I6 & MJD of photometric observation \\
18 & I12 & Spectroscopic target selection flag \\
19 & F6.2 & Period (hr); 0.00 is unknown period \\
20 & I3 & Reference \\
21 & A6 & Type of CV System \\
22 & A40 & Comments \\
\hline
\end{tabular}

(This table is available in its entirety in machine-readable and Virtual Observatory (VO) forms in the online journal. A portion is shown here for guidance regarding its form and content.)

Funding for the SDSS and SDSS-II has been provided by the Alfred P. Sloan Foundation, the Participating Institutions, the National Science Foundation, the U.S. Department of Energy, the National Aeronautics and Space Administration, the Japanese Monbukagakusho, the Max Planck Society, and the Higher Education Funding Council for England. The SDSS Web site is http://www.sdss.org/.

The SDSS is managed by the Astrophysical Research Consortium for the Participating Institutions. The Participating Institutions are the American Museum of Natural History, Astrophysical Institute Potsdam, University of Basel, University of Cambridge, Case Western Reserve University, University of Chicago, Drexel University, Fermilab, the Institute for Advanced Study, the Japan Participation Group, Johns Hopkins University, the Joint Institute for Nuclear Astrophysics, the Kavli Institute for Particle Astrophysics and Cosmology, the Korean Scientist Group, the Chinese Academy of Sciences (LAMOST), Los Alamos National Laboratory, the Max-PlanckInstitute for Astronomy (MPIA), the Max-Planck-Institute for Astrophysics (MPA), New Mexico State University, Ohio State University, University of Pittsburgh, University of Portsmouth, 
Princeton University, the United States Naval Observatory, and the University of Washington.

P.S. acknowledges support from NSF grants AST 0607840 and AST 1008734.

\section{REFERENCES}

Abazajian, K. N., Adelman-McCarthy, J. K., Agueros, M. A., et al. 2009, ApJS, 182,543 (DR7)

Abbott, T. M. C., Shafter, A. W., Wood, J. H., Tomaney, A. B., \& Haswell, C. A. 1990, PASP, 102, 558

Drake, A. J., Djorgovski, S. G., Mahabal, A., et al. 2009, ApJ, 696, 870

Feline, W. J., Dhillon, V. S., Marsh, T. R., Watson, C. A., \& Littlefair, S. P. 2005, MNRAS, 364, 1158

Fukugita, M., Ichikawa, T., Gunn, J. E., et al. 1996, AJ, 111, 1748

Gänsicke, B. T., Dillon, M., Southworth, J., et al. 2009, MNRAS, 397, 2170

Green, R. F., Schmidt, M., \& Liebert, J. 1986, ApJS, 61, 305

Gunn, J. E., Carr, M., Rockosi, C., et al. 1998, AJ, 116, 3040

Gunn, J. E., Siegmund, W. A., Mannery, E. J., et al. 2006, AJ, 131, 2332

Hagen, H. J., Groote, D., Engels, D., \& Reimers, D. 1995, A\&AS, 111, 195

Hillwig, T. C., Honeycutt, R. K., \& Robertson, J. W. 2000, PASP, 102, 558

Hogg, D. W., Finkbeiner, D. P., Schlegel, D. J., \& Gunn, J. E. 2001, AJ, 122, 2129

Howell, S. B., Nelson, L. A., \& Rappaport, S. 2001, ApJ, 550, 297

Ivezic, Ž., Lupton, R. H., Schlegel, D., et al. 2004, Astron. Nachr., 325, 583

Kato, T. 2010, vsnet-alert, 12026

Lupton, R. H., Gunn, J. E., Ivezic, Z., et al. 2001, in ASP Conf. Ser. 238, Astronomical Data Analysis Software and Systems X, ed. F. R. Harnden, Jr.,

F. A. Primini, \& H. E. Payne (San Francisco, CA: ASP), 269

Lupton, R. H., Gunn, J. E., \& Szalay, A. 1999, AJ, 118, 1406

Maehara, H. 2010a, vsnet-alert, 12020

Maehara, H. 2010b, vsnet-alert, 12025
Misselt, K. A., \& Shafter, A. W. 1995, AJ, 109, 1757

Olech, A., de Miguel, E., Otulakowska, M., et al. 2011, A\&A, 532, A64

Padmanabhan, N., Schlegel, D. J., Finkbeiner, D. P., et al. 2008, ApJ, 674, 1217

Pier, J. R., Munn, J. A., Hindsley, R. B., et al. 2003, AJ, 125, 1559

Richards, G. T., Fan, X., Newberg, H. J., et al. 2002, AJ, 123, 2945

Richter, G. A., \& Greiner, J. 1995, ASSL, 205, 177

Ringwald, F. A., Chase, D. W., \& Reynolds, D. S. 2005, PASP, 117, 1223

Ritter, H., \& Kolb, U. 2003, A\&A, 404, 301

Rodriguez-Gil, P., Gänsicke, B. T., Hagen, H.-J., et al. 2005, A\&A, 440, 701

Schmidt, G. D., Szkody, P., Vanlandingham, K. M., et al. 2005, ApJ, 630, 1037

Schneider, D. P., Hall, P. B., Richards, G. T., et al. 2007, AJ, 134, 102

Schwope, A. D., Brunner, H., Buckley, D., et al. 2002, A\&A, 396, 895

Smith, J. A., Lupton, R. H., Bernardi, M., et al. 2002, AJ, 123, 485

Stoughton, C., Lupton, R. H., Bernardi, M., et al. 2002, AJ, 123, 485

Szkody, P., Anderson, S. F., Agüeros, M., et al. 2002, AJ, 123, 430 (Paper I)

Szkody, P., Anderson, S. F., Hayden, M., et al. 2009, AJ, 137, 4011 (Paper VII)

Szkody, P., Fraser, O., Silvestri, N., et al. 2003, AJ, 126, 1499 (Paper II)

Szkody, P., Henden, A., Agüeros, M., et al. 2006, AJ, 131, 973 (Paper V)

Szkody, P., Henden, A., Fraser, O., et al. 2004, AJ, 128, 1882 (Paper III)

Szkody, P., Henden, A., Fraser, O. J., et al. 2005, AJ, 129, 2386 (Paper IV)

Szkody, P., Henden, A., Mannikko, L., et al. 2007, AJ, 134, 185 (Paper VI)

Thorstensen, J. R., Schwarz, R., Schwope, A. D., et al. 2009, PASP, 121, 465

Tucker, D., Kent, S., Richmond, M. W., et al. 2006, Astron. Nachr., 327, 821

Voges, W., Aschenbach, B., Boller, T., et al. 2000, IAU Circ., 7432

Voges, W., Aschenbach, B., Boller, Th., et al. 1999, A\&A, 349, 389

Warner, B. 1995, Cataclysmic Variable Stars (Cambridge: Cambridge Univ. Press)

Wenzel, W., Richter, G. A., Luthardt, R., \& Schwartz, R. 1995, IBVS, 4182, 1

Wickramasinghe, D. T., \& Ferrario, L. 2000, PASP, 112, 873

Wils, P., Gänsicke, B. T., Drake, A. J., \& Southworth, J. 2010, MNRAS, 402, 436

York, D. G., Adelman, J., Anderson, J. E., Jr., et al. 2000, AJ, 120, 1579 